\title{
Explorando los sentimientos de los padres, la familia y la sociedad a las personas con Síndrome de Down: Estudio observacional
}

\author{
Lourdes Huiracocha T. ${ }^{1}$, Carlos A. Almeida D. ${ }^{2}$, Karina Huiracocha ${ }^{3}{ }^{3}$, Andrea Arteaga H. ${ }^{2}$, Jorge \\ A Arteaga H. ${ }^{4}$, Paulina Barahona H. $^{2}$, Juan E Quezada H. \\ ${ }^{1}$ Facultad de Ciencias Médicas, Universidad de Cuenca, Av. 12 de Abril s/n, Cuenca, Ecuador. \\ ${ }^{2}$ Ministerio de Inclusión Económica y Social, Av. Remigio Crespo, Cuenca, Ecuador. \\ ${ }^{3}$ Facultad de Ciencias Médicas, Universidad del Azuay, Av. 24 de Mayo y Circunvalación, Cuenca, \\ Ecuador. \\ ${ }^{4}$ Facultad de Ciencias Médicas, Universidad Católica, Pío Bravo y Manuel Vega, Cuenca, Ecuador. \\ Autor para correspondencia: lourdes.huiracocha@ucuenca.edu.ec \\ Fecha de recepción: 18 de agosto 2013 - Fecha de aceptación: 16 de septiembre 2013
}

\section{RESUMEN}

Estudio cualitativo observacional que explora sobre los sentimientos y reacciones de los padres con hijos con Síndrome de Down (SD) a través de un grupo focal con 8 personas y de entrevistas semiestructuradas. Los niños participantes en la investigación pertenecieron a un Centro de Atención para personas con SD de la ciudad de Cuenca, Ecuador. La investigación identificó el significado cultural sobre las personas con SD, los sentimientos de los padres, el impacto en la familia y la comunicación de la noticia en el servicio de salud. El estudio reveló que las actitudes negativas con respecto a las personas con SD persisten. Adicionalmente, las entrevistas dieron luces sobre las respuestas de adaptación de los padres y de las familias. Por último, pero no menos importante, se reconoce la necesidad de mejorar la comunicación del personal de salud de acuerdo a los sentimientos y funcionamiento familiar para la aceptación y el cuidado de las personas con SD. Los resultados conducen a desarrollar programas de asesoramiento para las personas con SD, sus familias y para aumentar la conciencia y el apoyo social de la comunidad.

Palabras clave: Síndrome de Down, estudio cualitativo observacional, respuesta de los padres, reacción de la familia, estigma social.

\begin{abstract}
The paper presents the results of a qualitative observational study mapping the emotional responses of a focus group of 8 persons with children with Down Syndrome. The respondents also completed a semi-structured questionnaire. The children of the focus group all belong to the DS daycare centre of the city of Cuenca, Ecuador. The group interview and questionnaire addressed respectively the crossculturally stigma and discrimination versus persons with DS, the parent's feelings, the impact on the whole family, and the communication with the health care sector. The study revealed that in Cuenca city the negative attitude regarding DS persons still persists. Additionally, the survey shed light on the responses and adaption of parents and families. Last but not least it is recognized that improved communication with health care personnel, knowledgeable of the emotional attitude of parents and family functioning, will be beneficial for the acceptance and care-taking of DS persons. Findings leads to the necessity of developing counseling programs for children with DS and their families, and underscores the vital need to increase community awareness and social support.
\end{abstract}

Keywords: Down syndrome, qualitative observational study, parental response, family reaction, societal stigma. 


\section{INTRODUCCIÓN}

Las personas con síndrome de Down (SD) son portadoras de una alteración cromosómica en el par 21 (trisomomía, mosaicismo, traslocación), con fenotipo propio, déficit intelectual y malformaciones múltiples. La discapacidad en general se lleva a una dificultad para la realización de las actividades de la vida diaria cuyo nivel depende de las características individuales (comorbilidad), de la atención profesional interdisciplinaria y del apoyo que le brinde la familia y la comunidad (Bull y Comité de Genética, 2011). En la época actual la calidad de vida de las personas con SD ha mejorado trascendentalmente por los nuevos enfoques y prácticas en educación, salud, inclusión (Cabra, 2004). Sin embargo, cuando las familias tienen un hijo con SD experimentan una serie de sentimientos, reacciones acorde a: (i) el significado cultural de la sociedad y de la familia; (ii) la realidad de las personas con discapacidad en el Ecuador que confirma el significado cultural; (iii) el tipo de atención en el servicio de salud donde nace el niño o niña y como fue comunicada la noticia; y (iv) la dinámica familiar previa.

\subsection{El significado cultural de las personas con síndrome de Down}

El significado cultural son los diversos imaginarios, conceptos o visiones que las personas tienen frente a la discapacidad y que se expresan en actitudes, prácticas. El significado cultural de la discapacidad (Hernández, 2001) ha variado progresivamente yendo desde la concepción de dioses contrahechos (de un solo ojo, con mutilaciones, enanos), de inválidos para exterminio (cuando los pueblos eran nómadas o luego de las guerras) hasta bufones, mendigos, feos, incapacitados. En la edad media las personas con discapacidad (PCD) eran confinadas a cárceles de enfermos (hospicios, hospitales, subterráneos y otros), en el renacimiento con el cambio de las estructuras políticas, legales y científicas se empezó a visibilizarlas como seres que requieren ayuda, nace la rehabilitación dirigida únicamente al individuo. Foucault (1974-1975) destaca la dicotomía normal y anormal y se visibiliza a las PCD como seres humanos no normales que requieren acciones para normalizarlos. Finalmente, con los nuevos conceptos se da la denominación de Personas con Discapacidad buscando dignidad y calidad de vida mediante la atención integral y óptima (salud, educación, participación, inclusión) (Céspedes, 2010).

Existen diversas investigaciones que demuestran por un lado significados culturales que mantienen el estigma, la discriminación (Moreno y col., 2006; Díaz y Malpica, 2006) y otras en donde las familias expresan sentirse que tener una persona con SD ha sido una oportunidad para cambiar positivamente la vida (Wuang y Su, 2012). Las investigaciones realizadas en Estados Unidos (Pace $y$ col., 2010), revela que los adultos siguen teniendo actitudes negativas hacia las personas con SD porque la cuarta parte de los encuestados cree que los estudiantes con este síndrome deben ir a escuelas especiales. Casi el 30\% de los encuestados estuvo de acuerdo que la inclusión de las personas con SD en los centros educativos regulares distrae a los otros niños, el 18\% afirma que las personas con SD aumentan la posibilidad de accidentes en los lugares de trabajo. Las actitudes negativas también la tuvieron los jóvenes porque el $27 \%$ manifestó no estar dispuestos a trabajar en un proyecto de clase con estudiantes con SD y el $40 \%$ indicó no querer pasar tiempo fuera de la escuela con un estudiante con SD. Las mujeres encuestadas tanto adultas como jóvenes tienen mejores actitudes de aceptación que los hombres. Mientras que el estudio realizado por Skotko y col. (2011) a 2044 padres con hijos con SD revela que el $99 \%$ ama a su hijo o hija, el $97 \%$ se sienten orgullosos de ellos, el 79\% consideró que su actitud frente a la vida era más positiva a causa de ellos, el 5\% se sentía avergonzado por ellos y el $4 \%$ lamenta tenerlos, los padres señalan que el 95\% de sus hijos o hijas sin SD tienen buenas relaciones con sus hermanos con el síndrome. Según Skotko y col. (2011) la mayoría de los padres informan estar contentos con su decisión de tener a su hijo con SD e indican que son una gran fuente de amor y de orgullo.

\subsection{La realidad de las PCD en el Ecuador}

No hay datos específicos para las personas con SD pero el Consejo Nacional de Discapacidades de Ecuador (CONADIS, 2005) informa los siguientes datos que dan cuenta de la realidad de las PCD: (i) 
el $50 \%$ de las PCD están en el quintil 1 y 2 de pobreza; (ii) el $45 \%$ de las familias indican sentirse insatisfechos con la comunicación en los servicios de salud, las familias no son dirigidas ni incorporadas a un sistema con buena calidad de atención interprofesional, solo entre el 10 y el $30 \%$ de las personas con discapacidad alcanzan un servicio pero no todas las terapias; (iii) el $74 \%$ de la población con limitación grave no tiene las ayudas técnicas para desarrollar las habilidades adaptativas de escuchar, hablar, movilizarse y otras; y (iv) el 9\% de las PCD entre 5 y 9 años terminó la escuela y el $10.3 \%$ con discapacidades graves asistió a una escuela especial; (v) el $70 \%$ de la población con discapacidad indica ser discriminada en todos los campos; (vi) generalmente las PCD están confinadas en sus casas sin ningún plan de intervención y solo entre el 5 y el 30\% participan en alguna actividad social, religiosa o de asociación. La investigación realizada en los Centros de Desarrollo Infantil Urbanos de Cuenca en niños y niñas menores de 5 años de edad, indica que solo el $10 \%$ de estas instituciones integró a niños con discapacidad y que el $93 \%$ de ellos no tuvo ni diagnóstico ni plan de intervención ni adaptaciones curriculares (Huiracocha y col., 2012). Estos datos indican que la sociedad ecuatoriana es excluyente a pesar de la doctrina de derechos garantizada en las leyes y políticas públicas, realidad que marca más el significado cultural de discriminación por eso nadie desea tener un hijo o hija con SD.

\subsection{La atención y la comunicación de la noticia en el servicio de salud}

La Constitución Ecuatoriana del 2008 y el Código de la Niñez de la Adolescencia garantizan la atención con calidad e interdisciplinaria para el desarrollo óptimo y autónomo de las PCD (Asamblea Constituyente, 2008; Foro de la Niñez y Adolescencia, 2004), este derecho se sustenta en la innovación de las prácticas profesionales y de los servicios para hacerlos más eficientes, organizados, integrales, solidarios y cálidos (Peñafiel y col., 2003). Atención con calidad es también comunicar asertivamente la noticia, los mensajes, las directrices, de manera que las personas se sientan cómodas, seguras, confiadas, queridas y apoyadas (PROAPS, 2007). La comunicación a las familias sobre el diagnóstico del SD debe ser de forma honesta, cariñosa, positiva, acompañado del apoyo en salud y otros, porque permite a las familias llegar a aceptar, a adaptarse, a regularse; sin embargo, hay pocos datos científicos y poca instrucción en programas de formación sobre la mejor forma de trasmitir la noticia (Dent y Carey, 2006). En el 2009 investigadores realizaron una revisión sistemática de estudios desde 1960 a 2008 en la base de datos de Medline, PsychInfo y de otras bases académicas importantes averiguando el cómo los médicos deben comunicar la noticia del nacimiento de niños con SD y concluyeron que los padres prefieren recibir el diagnóstico juntos en una reunión con el obstetra y el pediatra, en un lugar privado e inmediatamente cuando el médico sospecha, los padres desean que se les informe sobre grupos de apoyo y los recursos locales (Skotko y col., 2009). El estudio etnográfico realizado en Medellín mediante entrevistas semiestructuradas a 20 madres y una abuela indica que los trabajadores de la salud trasmitieron la noticia del nacimiento de un niño con SD de forma fría y deshumanizada, desde una mirada de la discapacidad sin solución y esto no favoreció el encuentro de la madre con el hijo, creando incertidumbre sobre la visión de futuro (Bastidas y Alcaraz, 2011). Datos similares ofrece la investigación cualitativa realizada a 14 madres de habla española pero en sus resultados aparecen además: la información que reciben es incompleta, son explicadas desde las creencias religiosas y las madres se sienten excluidas de las decisiones médicas (Sheets y col., 2012). Otro estudio cualitativo realizado en Cuba desde julio de 2007 hasta abril de 2008 en 276 padres de niños con discapacidad menores de 15 años y en 136 profesionales identificó en el análisis que la falta de información sobre la discapacidad influenció en la calidad de atención en los servicios de salud porque el personal actuó subjetivamente más desde los temores que desde la experticia profesional (Rodriguez y col., 2009).

\subsection{La dinámica familiar, sentimientos y reacciones con el nacimiento de un niño con SD}

La familia inmersa en la sociedad desde el enfoque sistémico estructural (espacio cultural con sentidos simbólicos que construye la subjetividad de los individuos) e integrada por personas unidas emocionalmente y/o por lazos de sangre tiene dos funciones: (i) protección psicosocial (cuidado físico, afectividad, seguridad económica, educación y orientación) para lo cual cada uno de los miembros tiene roles y responsabilidades; y (ii) la acomodación a los cambios. Indudablemente el nacimiento de 
un niño o niña con SD implica aceptar una condición crónica en la que la familia tendrá que visualizar en toda su vida cotidiana la presencia de una persona con discapacidad. La familia debe gastar más energía y recursos económicos; la familia debe reorganizarse, autorregularse, debe permitir que los roles, las funciones, los valores de cada uno y de todos los miembros juntos se dirijan a la solución de las dificultades y a avizorar las oportunidades que conlleva tener un hijo o hija con discapacidad.

Cuando las familias han venido cumpliendo con sus funciones adecuadamente y reciben todos los apoyos para una atención integral y de calidad logran adaptarse positivamente y llegan a visibilizar las cualidades de las personas con SD, transforman sus creencias, expectativas hacia la oportunidad de aprender otra forma de vivir con dignidad y en solidaridad (Sauceda y Maldonado, 2003; Núñez, 2007). Sin embargo, la negación, el rechazo oculto, la frustración, la inadaptación al tener una persona en la familia con SD puede prevalecer cuando las familias no tienen ni la dinámica ni todas las condiciones óptimas porque: (i) no pueden cumplir las funciones de protección ni de adaptación; (ii) viven en una sociedad con significados culturales que conducen a hábitos y a costumbres que reproducen inequidades (Peñuela, 2012); (iii) conviven en el machismo que sobrecarga roles en las mujeres haciéndolas únicas responsables del cuidado de los hijos e hijas porque desde que se casan los hijos y el esposo son lo más importante (Larrañaga y col., 2008); (iv) asumen la religiosidad como factor que los culpabiliza o desculpabiliza, debiendo cargar la cruz hasta la muerte (Sánchez, 2009). Entonces las familias terminan ocultándolos y negándoles el derecho a la inclusión.

De todas formas, no importa como sea la familia, al recibir la noticia del nacimiento de un niño o niña con SD, los padres transitan por una gama de sentimientos y reacciones diversas, al inicio existe un gran impacto (shock o bloqueo), seguido de una fase de incredulidad. Los padres no quieren aceptar la inesperada noticia y suelen recurrir a la negación de esta realidad como mecanismo de defensa; posteriormente a la medida de que van asumiendo la realidad surge entre ellos los sentimientos de culpabilidad (acusaciones mutuas y propias de lo ocurrido) frustración y miedo a la opinión de los demás, incertidumbre sobre cómo cuidar al niño o niña. Se debaten al mismo tiempo entre la tristeza y la alegría, viven un período de duelo en el que necesitarán el máximo apoyo mutuo y de familiares, amigos, profesionales, asociaciones para llegar a la comprensión y asimilación de la discapacidad (Rogers y Dolva, 2002; Morales y López, 2006; Shelley y col, 2009).

El estudio cualitativo realizado en el año 2005 en Brasil mediante entrevistas semiestructuradas a 13 mujeres con hijos con SD entre otros resultados reporta que luego de recibir la noticia del nacimiento presentaron estrategias defensivas, disturbio de la autoestima, miedo, negación ineficaz (Nobrega y Oliveira, 2005). Otro estudio cualitativo en Brasil revela que las madres al recibir la noticia sienten desamparo con desesperanza, abandono y culpa. En la tentativa de reducir el desamparo, las mujeres buscan los poderes sobrenaturales, desarrollan actitudes de lucha en la búsqueda de medios que posibiliten la supervivencia y la inclusión de sus hijos. El futuro de ellas está entrelazado al de los hijos en una confirmación del ideal de familia perfecta (Tavares, 2004). En un estudio cualitativo de Londres, los investigadores al recoger las opiniones de 19 personas altamente comprometidas con la discapacidad (15 padres y 4 profesionales) concluye que criar a un hijo con SD es una experiencia que cambia la vida y obliga a las familias a reanalizar sus sistemas de creencias, valores, prioridades de manera que piensan de modo diferente sobre el niño, su papel como padres y el papel de la familia. Con el tiempo se asumen adaptaciones positivas, y se aprecian las contribuciones positivas que los hijos con discapacidad hacen a la familia y a la sociedad en su conjunto (King y col., 2006).

La investigación presentada en ese manuscrito tuvo como objetivos: (i) describir el significado cultural sobre las personas con SD; (ii) identificar el tipo de atención en el servicio de salud donde nace el niño o niña y como fue comunicada la noticia; y (iii) los sentimientos y reacciones de las familias al nacer un hijo o hija con SD. Con los resultados de la investigación se podrá tener mejores elementos para la elaboración de programas de intervención a los padres y familias de los niños y niñas con SD y para la sensibilización de los profesionales en miras de conseguir una comunicación asertiva. 


\section{METODOLOGÍA}

Un estudio cualitativo descriptivo, con enfoque teórico metodológico del interaccionismo simbólico (Sandoval, 2002) realizado de enero a abril del 2010 en un Centro de Atención Especializado para Personas con Síndrome de Down de la ciudad de Cuenca. Se realizó un grupo focal con 7 madres y un padre (ocho familias) con edades entre 28 a 40 años, todas las familias fueron nucleares. Se hicieron cuatro entrevistas que se seleccionaron por teoría y conveniencia. La edad de los niños y niñas fue de menos de 2 años ( 3 niños), de 6 años (1 niña) y una adolescente de 16 años. La última había migrado por diferentes instituciones y permaneció en casa durante los últimos 6 años sin asistir a ningún terapia hasta antes de ingresar a la institución estudiada. Las preguntas fueron elaboradas considerando cuatro existenciales básicos: (i) el lugar donde nació el niño o niña con síndrome de Down, hospital, clínica de la Ciudad de Cuenca y la forma como se dio la noticia (espacialidad); (ii) los sentimientos del padre, de la madre, de la familia al recibir la noticia y las reacciones pasivas, activas de enfado o activas (el cuerpo vivido); (iii) cuando ocurrió el nacimiento, las expresiones del sistema familiar, la red de relaciones (el tiempo vivido); (iv) la familia, el personal de salud en la atención, la comunicación (las relaciones humanas vividas).

Las categorías conceptuales empleadas fueron:

$\checkmark$ Significado cultural sobre las personas con SD: pensamientos, creencias, valores que generan en las personas sin discapacidad, actitudes de aceptación o de rechazo o prejuicios (estigma) o de exclusión o restricción de los derechos (discriminación) (CONADIS, 2005; Arnold, 1987).

$\checkmark$ Sentimientos: parte consciente de las emociones, expresiones con mayor intelectualidad fruto de un proceso subjetivo (memoria, asociación, introspección) y que conduce a las personas a adoptar conductas más racionales frente a los eventos internos y externos. Son sentimientos: (i) miedo: angustia frente a lo real y a lo imaginario. Aprehensión de que suceda algo contrario a lo que se desea. Paralización y falta de ánimo para seguir; (ii) aversión: oposición, disgusto, rechazo, resistencia; (iii) alegría: vivencia de satisfacción por haber recibido lo deseado o lo bueno inesperado, entusiasmo en hacer las cosas; (iv) ira: enojo contra algo o contra alguien que lleva a la persona a reaccionar fuertemente con llantos, gritos, protestas y otros; (v) tristeza: melancolía por situaciones difíciles de soportar, se expresan con llanto, silencio, alejamiento, retracción; (vi) dolor: malestar por algo o alguien que lastima hasta cambiar el ánimo; y (vi) otros (Belmonte, 2007).

$\checkmark$ Reacciones: patrón de acciones inmediatas y mediatas originadas de los sentimientos, la dinámica, la cultura frente a las personas con SD. Son: (i) reacciones pasivas: acciones que demuestran represión, inmersión, sin iniciativa y con necesidad de ser dirigidos; (ii) reacciones activas de enfado: conductas de llanto, grito, protesta, rechazo que no llevan a ninguna solución; y (iii) reacciones activas de resiliencia: actividades de búsqueda e instauración de soluciones para la atención de la persona con SD y de restitución de la dinámica familiar (Skotko y Canal, 2004; Bastidas y Alcaraz, 2011).

$\checkmark$ Comunicación del personal de salud: forma de trasmitir mensajes verbales y no verbales sobre el nacimiento del niño o niña con SD. Son (i) asertiva: trasmisión real de la noticia con conocimiento sobre las nuevas oportunidades en el diagnóstico e intervención y apoyo en el seguimiento de la atención; y (ii) no asertiva: cuando el personal solo trasmite la noticia y causa desconcierto en la madre, en el padre y en la familia, no hay un apoyo en la atención (PROAPS, 2007).

Aunque el equipo de investigadores estuvo constituido por 3 profesionales y 4 estudiantes de medicina, tanto en el grupo focal como en las entrevistas participaron los tres profesionales, el experto en terapia familiar hizo las preguntas, la educadora especial observaba y el pediatra hizo las anotaciones. Los participantes autorizaron las grabaciones. Las entrevistas se hicieron en un solo tiempo, los estudiantes ayudaron en la transcripción de los datos desde la grabadora digital, en los grupos de debates durante el análisis, en las revisiones bibliográficas y en la codificación. Durante el análisis se regresó a los entrevistados cuando fue necesario confirmar hallazgos y revisar algunos datos particulares con la intención de que ellos chequearan y corrigieran los errores de interpretación o completaran su participación. 
La reducción, preparación, el análisis de los datos cualitativos se hicieron manualmente: codificación descriptiva abierta, codificación axial o relacional y codificación selectiva. En el proceso de análisis, discusión del comportamiento y de las interpretaciones participaron los investigadores, y para la triangulación: una profesional psicóloga experta y una orientadora familiar. También se confrontó con varios expertos en el tema.

\section{RESULTADOS Y DISCUSIÓN}

\subsection{Imaginarios: entre pensares, creencias, visiones y realidades}

“... ¿Porque diosito manda estos niños? ... porque yo veía mucha gente que se burlaba... se burlaba y me daba rabia, era capaz de acercarme y decir -bueno usted de que se rie- si son personas igual que nosotros, son hijos de Dios ...".

En la identificación de los significados culturales, tal como lo documentan la mayoría de las investigaciones se encontró que las conceptualizaciones más frecuentes que las madres tienen sobre los niños y niñas con SD son: inválidos o deformes, de procedencia divina angelitos enviados al mundo para corregir, que necesitan mucho amor y dan amor, que son tiernos - buenos, que las mujeres añosas son las que los tienen, las familias indican que los hijos e hijas deben nacer sanos para que el hogar sea feliz. En las personas se conserva la visión de un Dios castigador, el que da y quita todo. Las pocas expresiones positivas que aparecen en las entrevistas son en personas que se han actualizado en los nuevos paradigmas de la diversidad, de la inclusión, o como en el caso del padre de la adolescente con SD de 16 años, que ya ha conocido las capacidades de estas personas (Skotko $y$ col., 2011).

\subsection{El momento, la atención, la noticia: la comunicación por parte del personal de salud y otros}

“.... entonces él lo que hizo es acabarme de un... -pausa- me acabó, me mató ya ... con las palabras groseras como él dijo, y no lloré porque ...".

Cuando se leen las entrevistas sorprende como suceden los eventos en las áreas de salud, de educación, lo técnico se divorcia de lo cálido y humano. Con naturalidad las madres cuentan lo sucedido en la atención en diferentes circunstancias:

- Actitudes prepotentes del médico, improvisación en el momento de la atención: “... me dijo así ... la niña es down ... yo le dije -pero como usted puede decir si usted .... no, es que yo tengo ojo clínico - y todo así ... de una manera bien déspota, de una manera sin corazón".

- La noticia es dada por personal de salud lejano a la madre y no por el médico de confianza: “... se acercaron un grupo de médicos ... dicen: sabe que su guagua tiene $S D$... tenemos que decirle la verdad".

- El dar anticipadamente criterios a la madre sin enterarse bien del caso: "Yo no sé si es que él vio o se hizo, yo no sé ... y me dice: es un lindo varón, está sanito ...".

- La ausencia de apoyo profesional, el personal de salud no indica los procesos ni los derechos a la atención integral de esos niños y niñas: “... señora mejor váyase a la casa porque aquí usted se estresa, nos estresa a nosotros y a la niña también - se ponía molesta ...".

- La comunicación castigadora: "si no aceptas a tú hijo entonces regálelo".

- La trasmisión no cálida de la noticia: “... porque yo creo que ellos deberían prepararse un poco más en ... en cómo llegar a la gente, como no herir tanto ...”. 
- La tardanza en la noticia: “... apenas me sacaron de la barriga, dijeron véale rápido y se llevaron ... después ... que sería la una y media había cambio de enfermeras, decían:-espere que venga el otro turno- ...".

- A esto se suma la comunicación no asertiva de los religiosos que inculpan y obligan a las madres a aceptar a sus hijos e hijas sin un proceso de intervención psicológica: -veras, yo te digo una cosa, voz no te eches la culpa que porque tu edad, que por qué ... Dios te mandó, él es un ángel, y verás que él te va a dar muchas bendiciones, y yo te digo una cosa: si tú le aceptas el guagua va a vivir y si tú ahorita dices no, Dios te va a quitar, y se va a llevar y tú te vas a quedar sin hijo y después el arrepentimiento ....".

- Tampoco hay una comunicación asertiva de las instancias educativas porque se basa en exigencias que son imposibles de cumplir y que a la larga imposibilitan la recepción de ayuda.

- Los familiares que ocultan información para evitar el sufrimiento de la madre pero que realmente causa más angustia: “... mi esposo, y mi cuñada dice .... ella ha estado ya con el bebé pero no quería decirme nada ...”.

- Se encuentran pocos casos de comunicación asertiva que motivan a creer en un futuro positivo: “... la doctora me decía: hoy la visión de la gente ha cambiado ... e incluso hay muchos niños que gracias a las terapias se están incluyendo dentro de una educación regular ...".

Los datos de la investigación concuerdan con los de la mayoría de las investigaciones (Dent y Carey, 2006; Skotko y col., 2009; Bastidas y Alcaraz, 2011; Sheets y col., 2012; Rodriguez y col., 2009) y con el estudio cualitativo exploratorio realizado en Bogotá en el 2008 en el que describe que las madres encuentran un trato ambivalente, excluyente por parte del personal de salud y reclaman reforma en la atención (Suárez y col., 2009). Otro estudio cuanti cualitativo del año 2004, realizado en España a 467 madres de niños con SD, a través de una encuesta sobre los servicios de apoyo postanatal que recibieron inmediatamente después del diagnóstico, las madres dijeron sentirse ansiosas, asustadas, culpables, enfadadas, y en casos excepcionales con deseos de suicidarse. Según la mayoría de las madres, los médicos no portaron información suficiente sobre el SD, y rara vez les dieron material escrito o les aconsejaron relacionarse con grupos de apoyo organizados por padres (Skotko y Canal, 2004).

\subsection{Los sentimientos y reacciones frente a la noticia del nacimiento}

“... yo digo mi hijo, no, no, no ha de ser mi hijo, usted está equivocada, digo porque el doctor que me mostró no, no... no puede ser mi hijo -una pausa- ... digo ustedes están equivocados no ve que hay tanto guagua que nace aqui ...".

Los sentimientos y reacciones son básicamente trasmitidos por la madre, porque en las narraciones, ellas no mencionan al esposo; incluso en el apoyo emocional, son otras las personas que están cerca. Se nota un gran silencio, una enorme distancia o un gran replegamiento de los sentimientos del padre, es decir se reproduce el patrón de comportamiento del hombre definido por los roles de género: no expresión de sentimientos, no llanto, distante del cuidado de los niños y niñas, externo al hogar. Cuando se leen las entrevistas se evidencia que la mayoría de las mujeres tienen embarazo y parto de alto riesgo pero a ninguna se le da la atención que requiere. Las mujeres entran asustadas a dar a luz y luego el personal de salud conociendo que los niños tienen SD, demoran la entrega del bebé a las madres respectivas. No hay procesos que les prepare o les atienda psicológicamente, ni el médico ginecólogo, ni el pediatra comunican la noticia, lo hacen las enfermeras o los médicos residentes durante las visitas de sala. Esto aumenta la ansiedad, el miedo y las mujeres tienen fuertes sentimientos de negación. A una de las madres le sedan y con palabras condenatorias le obligan a aceptar a su hijo. La madre es juzgada por un sacerdote, traído para consolar, pero que condena a la muerte de la madre o del niño si no acepta al hijo con SD. Estas circunstancias desencadenan una serie de sentimientos de dolor, miedo, tristeza, adversidad. La dimensión subjetiva de las madres las inhibe para actuar y analizar posibles alternativas de solución, desconcierto al percibir la situación como 
inesperada, lo que las desorienta y confunde. Las madres interpretan las condiciones de sus hijos como de fragilidad por lo que emprenden acciones de sobreprotección existiendo sentimientos ocultos de inconformidad ya que la debilidad que ven en su hijo representa su propia fragilidad. Aparece la madre como la figura primaria de cuidado reproduciendo las estructuras sociales y los roles de género, en donde la mujer es la que mejor se adapta y asume las funciones familiares para que su hogar no se desbarate. Al proceso afectivo inicial, luego del impacto, las madres le dan una interpretación cognitiva y espiritual (la procedencia divina de los niños y niñas con SD) que se centra en la realidad de manera que se inicia un proceso de aceptación que les permite asumir la situación de una manera más adaptativa visualizando mejores alternativas de acción que respondan a las características actuales de su hijo y les permitan estabilizar su estado psicoemocional (Dent y Carey, 2006; Rodriguez y col., 2009).

Las reacciones que mayormente aparecen son: (i) activas de resiliencia; (ii) activas de enfado, solidaridad y exigibilidad de derechos, y (iii) pasivas de resignación, auto marginación: “... dejé de ir a un gimnasio donde yo asistía todas las mañanas, he dejado de visitar a mis parientes, he dejado de salir ... solo voy a vivir cada día con mi hija y voy a hacer todo lo que esté en mis manos". Dentro de las activas de resiliencia de la madre están: (i) aceptar a su hijo y observar cualidades; (ii) no esconder a su hijo; (iii) dar cuidado y buscar la atención especializada; (iv) no entregarse a la depresión; y (v) buscar la protección legal, de defensa contra la sociedad que discrimina a los diferentes. Estos datos coinciden con investigaciones anteriores sobre la reacción inmediata de la madre en el momento que los niños nacen (Rogers y Dolva, 2002; Morales y López, 2006; Shelley y col, 2009).

\subsection{La familia frente a la noticia}

“... Ella ha cambiado la vida de todos nosotros, porque mis hijos ... hasta aceptar fue un desbarajuste, hasta ... acoplarnos a los nuevos cargos, los nuevos roles, lo que nos tocaba hacer a cada uno, pero ahora veo que ya la cosa esta mejor, me colaboran, me ayudan ...".

El nacimiento de un hijo con SD implica la transformación del círculo familiar, que lleva a una sobrecarga materna o paterna que puede afectar el vínculo conyugal y el vínculo paterno filial. En muchos casos podrá funcionar como un elemento de fractura y ruptura de la familia sobre todo si ya existían conflictos previos, pero también el hijo con discapacidad puede funcionar como un factor de unión y fortalecimiento mayor del vínculo, fundamentalmente en aquellos casos en los que la pareja tenía buenas bases en su constitución (Sauceda y Maldonado, 2003; Núñez, 2007). En la historia de nuestras familias entrevistadas se evidencia con el silencio. La no conciencia sobre las funciones y roles de todos y de cada uno porque repiten el patrón social: la madre es el eje espiritual, la responsable del cuidado de los hijos, de las tareas de casa; el padre es para el trabajo y se mantiene distante de cosas de mujeres (sentimientos, roles caseros); los hijos están para estudiar y solo cuando hay tiempo ayudan a mamá. Por estas razones cuando nace el hijo o hija con SD el mundo se transforma para la madre y los otros miembros solo acompañan en el dolor. Las mujeres de todas las entrevistadas mencionan que desde el nacimiento del niño o niña hubo mejora en las relaciones familiares, los hijos, el esposo se aproximaron pero es porque las mujeres se atribuyen casi todas las responsabilidades del cuidado reproduciendo los estereotipos femeninos de maternidad y cuidado asignados por el género (Quintero, 2007).

“... supóngase mi esposo trabaja, él es empleado, todos los demás están ocupados, entonces prácticamente yo soy la que le cuida y todo... porque él es mi hijo ...”.

En tres de las cuatro entrevistas el padre aparece silencioso en sus actuaciones durante la noticia y luego en la búsqueda de alternativas de atención para su hijo o hija. El resto de la familia (tíos, primos, abuelos) da consuelo pero no se involucra en la búsqueda de alternativas. En general, todas las familias deben hacer esfuerzos extras por conseguir mayor ingreso económico para pagar las terapias 
del hijo o hija, existen privaciones no solo de dinero sino de tiempo libre para el disfrute y el descanso. Las familias pasan constantemente de la tristeza a la alegría y viceversa como reflejo de la lucha constante por superar los problemas. Estas circunstancias ocasionan desconfianza, restricción de la participación en espacios sociales (Rogers y Dolva, 2002; Morales y López, 2006; Shelley y col, 2009).

\section{CONCLUSIONES}

Persiste el concepto negativo que se tiene sobre las personas con SD, los significados de estigma y discriminación adquiridos histórica y culturalmente influyen en las emociones, sentimientos y reacciones de la madre y de la familia que inicialmente son de dolor, de culpa, de miedo, frustración, negación. El personal de salud no atiende con calidad ni trasmite asertivamente la noticia. Las familias con el nacimiento de un niño o niña con SD ingresan en un estado de emergencia que cambia la dinámica que sobrecarga roles en la mujer. La madre progresivamente acepta y asume reacciones resilientes para buscar atención en salud, en educación, para ofrecer mejor calidad de vida al hijo o hija con SD. Con el tiempo los padres experimentan cambios en el modo de ver a sus hijos, a sí mismos y al mundo, encontrando en sus hijos e hijas con SD un amplio abanico de cualidades y la motivación para realizar transformaciones personales: paciencia, amor, compasión, tolerancia; mejoría en las relaciones con los miembros de la familia y con los demás; reforzamiento de las convicciones espirituales y religiosas; habilidad para centrarse en el presente; mayor apreciación de las cosas pequeñas y sencillas de la vida.

El estudio permite recomendar al personal de salud mejoramiento en la comunicación y atención de los niños y niñas con SD y sus familias, expresando sus competencias con el ejercicio de valores y ética que permita a los demás aceptar la diversidad y el reconocimiento de expresiones genéticas y de capacidades diferentes.

\section{AGRADECIMIENTOS}

Los autores agradecen al Centro de Desarrollo Integral para las Personas con Síndrome de Down (CEDIN DOWN), proyecto de la Fundación Huiracocha Tutivén, a las maestras, a los padres de familia, a los niños y niñas que para el acceso a la información y datos históricos han permitido la realización de la investigación.

\section{BIBLIOGRAFÍA}

Arnold, M., 1987. Exposición crítica teóricas de la Antropología cognitiva. Revista Chilena de Antropología, 6, 13-25. Descargado de http://www.revistas.uchile.cl/index.php/RCA/article/ viewFile/17622/18386 el 10 de agosto del 2009.

Asamblea Constituyente, 2008. Constitución 2008. Ministerio de Gobierno, Policía y Cultos. República del Ecuador, 24-25, 33-43.

Bastidas. M, G.M. Alcaraz, 2011. Comunicación de la noticia del nacimiento de un niño o niña con Síndrome de Down: el efecto de una predicción desalentadora. Rev. Fac. Nac. Salud Pública, 29, 17-24.

Belmonte, C., 2007. Emociones y cerebro. Rev. R. Acad. Cienc. Exact. Fis. Nat., 101(1), 59-68. Descargado de http://www.rac.es/ficheros/doc/00472.pdf el 10 de agosto del 2009.

Bull, M.J. y Comité de Genética, 2011. Clinical Report- Health supervision for children with Down Syndrome. Pediatrics, 128(2), 393-407. 
Cabra de Luna, M.A, 2004. Discapacidad y aspectos sociales: la igualdad de oportunidades, la no discriminación y la accesibilidad universal como ejes de una nueva política a favor de las personas con discapacidad y sus familias. Algunas consideraciones en materia de protección social. Revista del Ministerio de Trabajo y Asuntos sociales, 50, 21-46. Descargado de http://dialnet.unirioja.es/servlet/articulo?codigo=897754 el 13 de agosto del 2013.

Céspedes, M., 2010. La nueva cultura de la discapacidad y los modelos de rehabilitación (Spanish). Aquichan [serial on the Internet]. (2005, Oct), [cited February 16, 2010]; 5(1), 108-113. Available from: Academic Search Complete. Descargado de http://search.ebscohost.com/login.aspx? direct $=$ true $\& d b=a 9 h \& A N=21501715 \& a m p ;$ lang $=e s \&$ site $=$ ehost-live el 10 de febrero del 2010.

CONADIS, 2005. Discapacidad en cifras. Capítulo: Actitudes frente a la discapacidad (1 disco compacto). Consejo Nacional de Discapacidades, Instituto Nacional de Estadísticas y Censos del Ecuador.Díaz, J., C. Malpica, 2006. Representaciones sociales de la discapacidad. (Spanish). Investigación en Salud [serial on the Internet]. (2006, Dec), [cited February 16, 2010]; 8(3), 158164. Available from: Academic Search Complete. Descargado de http://search.ebscohost.com/ login.aspx? direct $=$ true $\& d b=a 9 h \& A N=24572676 \& a m p ;$ lang $=e s \&$ site $=$ ehost-live el 14 de febrero del 2010.

Díaz, J., C. Malpica, 2006. Representaciones sociales de la discapacidad. (Spanish). Investigación en Salud [serial on the Internet]. (2006, Dec), [cited February 16, 2010]; 8(3), 158-164. Available from: Academic Search Complete. Descargado de http://search.ebscohost.com/login.aspx?direct $=t r u e \& d b=a 9 h \& A N=24572676 \&$ amp; lang $=$ es\&site $=$ ehost-live el 14 de febrero del 2010.

Dent, K., J.C. Carey, 2006. Breaking difficult news in a newborn setting: Down síndrome as a paradigm. Am. J. Med. Genet. C. Semin. Med. Genet., 142C(3), 173-9.

Foro por la Niñez y Adolescencia, 2009. Análisis del Código de la Niñez y Adolescencia del Ecuador. Descargado de: http://www.revistajuridicaonline.com/index.php?option=com_content\&task=view \&id=58\&Itemid $=27$ el 14 de aogsto del 2013.

Hernández, R., 2001. Antropología de la discapacidad. Descargado de: http://www.peritajemedicoforense.com/RHERNADEZ.htm el 13 de agosto del 2013.

Huiracocha, L., G. Robalino, M.S. Huiracocha, J.L. García, C.G. Pazán, A. Angulo, C.A. Almeida, 2012. El desarrollo psicomotor y la calidad de la atención temprana. Maskana, 3(2), 31-44.

King, G., L. Zwaigenbaum, S. King, D. Baxter, P. Rosenbaum, A. Bates, 2006. Cambios en los sistemas de convicciones, valores de las familias de niños con autismo y síndrome de Down. Revista Síndrome de Down, 51-58. Descargado de http://www.downcantabria.com/revistapdf/ 89/51-58.pdf el 27 de noviembre del 2009.

Larrañaga, I., U. Martín, A. Bacigalupe, J.M. Begiristáin, M.J. Valderrama, B. Arregi, 2008. Impacto del cuidado informal en la salud y la calidad de vida de las personas cuidadoras: Análisis de las

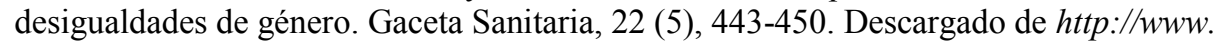
sciencedirect.com/science/article/pii/S0213911108724191 el 11 de agosto del 2013.

Morales, M.G.E., R.E.O. López, 2006. El Síndrome de Down y su mundo emocional. Editorial Trilla, México.

Moreno, F.M, M. Rodríguez, M. Duque, L. Ramírez, O. Pardo, 2006. ¿Qué significa la discapacidad? (Spanish). Aquichan [serial on the Internet]. (2006, Oct), [cited February 16, 2010]; 6(1), 78-91. Available from: Academic Search Complete. Descargado de http://search.ebscohost.com/ login.aspx?direct $=$ true $\& d b=a 9 h \& A N=23438098 \& a m p ;$ lang $=e s \&$ site $=$ ehost-live el 13 de Febrero del 2010.

Nobrega, A, M.V. Oliveira, 2005. Problemas de adaptación psicosocial de madres de niños portadores de síndrome de Down. Cultura de Cuidados, 17, 68-73.

Núñez, A., 2007. Familia y Discapacidad: de la vida cotidiana a la teoría. Primera edición. Editorial LUGAR, Buenos Aires.

Pace, J.E, M. Shin, S.A. Rasmussen, 2010. Understanding attitudes toward people with Down syndrome. Am. J. Med. Genet. A., 152A(9), 2185-92. 
Peñafiel, F., A. Hernández, A. Chacón, 2003. Atención temprana. Enseñanza, 21, 245-274.

Peñuela, J.R.J., 2012.Teletones en Chile y Colombia: De la Discapacidad como caridad, y la Caridad como negocio. Observatorio de la Economía Latinoamericana, Grupo Eumed.net (Universidad de Málaga). Descargado de http://scholar.googleusercontent.com/scholar? $q=$ cache:_EOPDfG9 hfwJ:scholar.google.com/+CARIDAD $+Y+D I S C A P A C I D A D \& h l=e s \& a s \_s d t=0,5$ el 13 de agosto del 2013.

PROAPS (Programa de Reforma de la Atención Primaria de Salud), 2007. Manual de Comunicación para la Salud. Descargado de http://www.anep.edu.uy/documentos/herramientas.pdf en julio del 2013, pág. 15-35.

Quintero, M., 2007. Diccionario Especializado en Familia y Género. Editorial Lumen, México.

Rodríguez, Y., A. Martínez, O. Álvarez, A. Socarrás, D. Marrero, 2009. Estrategia para mejorar la calidad de la atención al niño con discapacidad. MEDISAN, 13(2). Descargado de $h t t p: / / b v s$. sld.cu/revistas/san/vol13_2_09/san06209.htm el 26 de Noviembre del 2009.

Rogers, Ch., G. Dolva, 2002. Nuestra hija tiene síndrome de Down. Tercera Edición. Editorial Paidos, Barcelona.

Sánchez, B., 2009. Bienestar espiritual en personas con y sin discapacidad. Aquichán, 9(1), 8-22. Descargado de http://www.scielo.org.co/scielo.php?script=sci_arttext\&pid=S1657-59972009 $000100002 \& \operatorname{lng}=e n \& n r m=i s o$ el 10 de agosto del 2013.

Sandoval, C., 2002. Investigación cualitativa. Programa de Especialización en Teoría, Métodos y Técnicas de Investigación Social. ARFO Editores, Bogotá. Descargado de http://epistemologiadoctoradounermb.bligoo.es/media/users/16/812365/files/142090/INVESTIGACION_CUALITATI VA.pdf el 12 de agosto del 2009.

Sauceda, J., M. Maldonado, 2003. La familia: su dinámica y tratamiento. Organización Panamericana de la Salud. Editorial IMSS, Washington DC, EE.UU.

Sheets, K.M., B.J. Baty, J.C. Vásquez, J.C. Carey, W.L. Hobson, 2012. Breaking difficult news in a cross- cultural setting: A qualitative study about Latino mothers of children with Down syndrome. J. Genet. Couns., 21(4), 582-90.

Shelley, M.C., V.D. Veek, V. Kraaij, N. Garnefski, 2009. Down or up? Explaining positive and negative emotions in parents of children with Down's syndrome: Goals, cognitive coping, and resources. J. Intellect. Dev. Disabil., 34(3), 216-229.

Suárez, F., A. Ordoñez, M. Macheta, 2009. Percepción de las madres con hijos afectados por malformaciones congénitas mayores: necesidad de desarrollo de un sistema de atención apropiado. Estudio cualitativo de grupos focales. Descargado de $h t t p: / / w w w . b i o l i n e . o r g . b r / p d f ?$ rc09011 el 12 de agosto del 2013.

Skotko, B.G., R. Canal, 2004. Apoyo postnatal para madres de niños con síndrome de Down. Revista Síndrome de Down, 21, 54-71. Descargado de http://www.downcantabria.com/documentos/ Apoyo_postnatal.pdf el 12 de agosto del 2013.

Skotko, B.G., G.T. Capone, P.S. Kishnan, 2009. Postnatal diagnosis of Down syndrome: synthesis of the evidence on how best to deliver the news. Pediatrics, 124, 751-758.

Skotko, B.G, S.P. Levine, R. Goldstein, 2011. Having a son or daughter with Down syndrome: perspectives from mothers and fathers. Am. J. Med. Genet. A., 155A(10), 2335-47.

Tavares, A., 2004. La subjetividad de la mujer madre Down en el Nordeste Brasileño, una vida severina. Descargado de http://www.cibernetia.com/tesis_es/PSICOLOGIA/PSICOLOGIA_DE_ LA_ANORMALIDAD/DEFICIENCIA_MENTAL/1\#sthash.CPyBvYpb.dpuf el 14 de agosto del 2013.

Wuang, Y., C.Y. Su, 2012. Patterns of participation and enjoyment in adolescentes with Down síndrome. Res. Dev. Disabil., 33(3), 641-8. 